\title{
ВОЗМОЖНОСТИ МАНУАЛЬНОЙ ТЕРАПИИ В КОРРЕКЦИИ СКОЛИОЗА У ДЕТЕЙ СО СПИНАЛЬНЫМИ МЫШЕЧНЫМИ АТРОФИЯМИ
}

Спинальная мышечная атрофия (CMA, OMIM 253300) представляет собой нейродегенеративное заболевание с вовлечением двигательных нейронов спинного мозга, дебютом преимущественно в раннем детском возрасте с вариацией частоты заболеваемости - от 1 на 6000 до 1 на 10000 новорожденных [1-3]. Молекулярногенетические механизмы связаны с дефектом гена выживаемости мотонейрона (SMN 1), локализующегося в области 5q11.2-q13.3 [4, 5]. Клинический паттерн всех типов СМА включает симптомокомплекс вялого пареза (миогенная атрофия и слабость), приводящего к стойкому ограничению двигательной функции конечностей с различной степенью тяжести тетрапареза, что обусловлено фенотипическими факторами [2, 3]. Прогрессирующая мышечная слабость приводит к ограничению двигательной функции туловища ребенка и сопровождается развитием сколиотической деформации позвоночника различной степени выраженности, что во многом определяет тяжесть клинического течения и витальный прогноз заболевания [6, 7]. Комплекс реабилитационных мероприятий у детей СМА направлен прежде всего на коррекцию двигательных нарушений через активацию компенсаторно-приспособительных возможностей организма и базируется на индивидуальной оценке характера и степени выраженности двигательных нарушений, что позволяет составлять персонифицированную программу реабилитационных мероприятий, включая методы мануальной коррекции вертеброгенных нарушений.

\section{ЦЕЛ В}

Оценить возможности мануальной коррекции сколиотических деформаций позвоночника у детей СМА типа 2.

\section{МАТЕРИАЛ И МЕТОДЫ}

На базе Республиканского клинического центра нейрореабилитации (Донецк) обследованы 54 ребенка детей со СМА 2 типа, включая
36 мальчиков (66,7\%) и 18 девочек 29 (33,3\%). В рамках генетической верификации проводилось исследование генотипа мутации в ФГБН «Медико-генетический научный центр» (Москва), Медико-генетическом центре «Геномед» (Ростов), Институте молекулярной биологии и генетики НАНУ (Киев). Дизайн работы - проспективное когортное исследование, способ формирования выборки - стратифицированный (простая случайная выборка).

Родители или законные представители пациентов были ознакомлены и информированы о целях, характере, диагностических и лечебных процедурах, а также о возможных неблагоприятных эффектах лечения и дали добровольное информированное согласие.

Критерии включения в исследование: возраст 3-7 лет, генетически верифицированная форма проксимальной СМА с аутосомно-рецессивным типом наследования, соответствие клиническому фенотипу промежуточной формы (2 тип).

Критерии исключения: наличие тяжелой соматической патологии, отказ родителей или законных представителей от исследования.

Средний возраст пациентов на начало иссле-

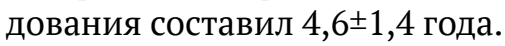

В исследовании пациенты, в зависимости от проводимой терапии, были разделены на 2 группы:

- основную группу составили 35 детей (64,8\%), у которых в программу реабилитационных мероприятий были включены методы мануального воздействия на позвоночник;

- в группу сравнения вошли 19 детей (35,3\%), которым мануальная терапия не проводилась.

Меньшее количество детей в группе сравнения обусловлено стремлением оказать эффективную помощь большему количеству пациентов ввиду орфанности заболевания.

(c) М.Р. Шаймурзин, И.С. Луцкий, О.С. Евтушенко,

М.В. Ефименко, 2020

(c) Университетская Клиника, 2020 
Пациенты обеих групп получали массаж по общепринятой методике.

Пациенты основной группы и группы сравнения проходили тестирование по международной шкале оценки функциональных возможностей Хаммерсмит (RHS) $[8,9]$.

Для оценки деформации позвоночного столба применялся рентгенологический метод исследования (Мобильный цифровой рентгеновский аппарат AGFA HealthCare DX-D 100 (Германия)) с последующим анализом величин угла боковой девиации по Коббу.

Контрольная оценка данных физикального и ренттгенологического исследования фиксировалась в установленные временные отметки заболевания («исходные данные», «3 года»).

Для коррекции рефлекторно-тонических нарушений, возникающих при различных деформациях позвоночного столба, проводились курсы мануальной терапии с применением приемов мобилизации и постизометрической релаксации (ПИР) [10]:

1) Мобилизация шейно-грудного перехода.

Использовался прием латерофлексии в положении ребенка лежа на спине с одновременной тракцией шейно-грудного перехода и проработкой мягких тканей путем их растягивания с одновременной фиксацией второй рукой сверху противоположного плеча пациента.

2) Мобилизация ребер

Манипуляция проводилась в положении пациента лежа на боку с заведенной за голову рукой. Врач становился сзади, одной рукой захватывал руку больного за плечо и во время выполняемого выдоха другой рукой давил вниз, одновременно создавая этой рукой сопротивление путем фиксации ладонью и разведенными большим и указательным пальцами соответствующих ребер.

3) Мобилизация грудного отдела с помощью давления на поперечные отростки сверху вниз.

Прием проводился в положении пациента лежа на животе с вытянутыми вдоль туловища руками и повернутой в сторону головой. Осуществлялся во время выдоха больного посредством давления скрещенными выпрямленными в локтевых суставах руками. Давление производилось сверху вниз на поперечные отростки верхнего и нижнего позвонков одновременно справа и слева от позвоночника приложенными к поперечным отросткам разогнутыми в области гороховидных костей ладонями. При этом давление оказывалось плечевым поясом.

4) Мобилизация поясничного отдела.

Прием осуществлялся в положении лежа на животе. При этом согнутая в коленном суставе нога использовалась в качестве рычага. Боль- шой палец одной руки врач устанавливал на остистый отросток. Ладонью второй руки захватывал ногу больного и отводил ее до тех пор, пока не ощущал движение позвонка под большим пальцем. Мобилизация осуществлялась одновременным давлением на остистый отросток и смещением ноги больного.

5) ПИР ротаторов позвоночника.

Исходное положение врача - стоя, сбоку от пациента, лицом к нему. Одно предплечье врач фиксировал на гребне подвздошной кости ребенка, другое - в подмышечной впадине. Кисти рук фиксировали сегмент в поясничном отделе позвоночника. Врач производил ротацию в поясничном отделе позвоночника за счет движения его рук в противоположном направлении.

Статистическая обработка данных выполнена с использованием пакетов прикладных программ Statistica 10 и SAS JMP 11. Анализ динамики показателей производился на основе непараметрических критериев Вилкоксона (для зависимых групп) и Манна-Уитни (для независимых групп). Уровень статистической значимости был зафиксирован на уровне вероятности ошибки 0.05 .

\section{РЕЗУЛЬТАТЫ И ОБСУЖДЕНИЕ}

Согласно рекомендациям экспертов Европейского нейромышечного консорциума, Европейского общества спинальных мышечных атрофий установление диагноза СМА должно сопровождаться проведением молекулярногенетических исследований, которые позволяют обнаружить мутации SMN гена, локализующиеся в коротком плече 5 хромосомы [2, 7]. У всех пациентов, принимавших участие в исследовании, диагноз верифицирован генетически. Полученные результаты свидетельствуют о значительном преобладании детей с гомозиготной делецией (конверсией) экзона 7 и/или экзона 8 SMN1, которая обнаружена у 51 ребенка. У 3 пациентов обнаружена делеция на одной аллели и внутригенная мутация на другой (таблица 1).

В основе клинической классификации проксимальных форм СМА лежит возраст дебюта заболевания и уровень максимальной двигательной функции $[11,12]$. Для каждого из трех клинических СМА характерно наличие определенного числа копий гена SMN2, что является надежным биомаркёром, подтверждающим фенотипический вариант СМА. Так, для СМА типа 1 число копий гена SMN2 варьирует в пределах 1 - 2. У пациентов СМА типа 2 количество копий составляет от 2 до 3 . Наибольший показатель по числу копий SMN2 у детей CMA типа 3 - он находится в пределах от 3 до 5 [2, 4-6, 13]. 
Подсчёт количества копий гена SMN2 у участников исследования показал, что преобладали пациенты с тремя повторами указанного гена - их было 46 (85,2\%). Количество больных, у которых обнаружено по четыре копии SMN2 гена, составило 5 (10,9\%) детей, 2 копии гена имели 3 (3,9\%) пациента (табл. 1.). Полученное распределение числа копий SMN2 гена соответствовало литературным данным для проксимальных СМА типа 2.

Одним из проявлений слабости аксиальной группы мышц является формирование сколиотической деформации позвоночного столба. Для определения степени сколиоза проводили рентгенологическое исследование позвоночника в прямой и боковой проекциях в вертикальном положении (сидя, если ребенок сидячий, и стоя - у амбулаторных больных). Использовали стандартную методику измерения искривления позвоночника по Коббу (угол Кобба) [14, 15]. Тяжесть сколиоза оценивали следующим образом: при величине угла бокового отклонения не более $10^{\circ}$ констатировался сколиоз 1 степени, от $10^{\circ}$ до $25^{\circ}$ - сколиоз 2 степени, рентгенологическая величина деформационного угла от $26^{\circ}$ до $50^{\circ}$ расценивалась как 3 степень сколиоза, показатели угла боковой девиации более $50^{\circ}$ свидетельствовали в пользу сколиоза 4 степени.

Распределение пациентов в основной группе и группе сравнения в начале исследования было следующим: основная группа - 29 (82,9\%) детей со сколиозом 1 степени, $6(17,1 \%)$ пациентов - сколиоз 2 степени, в группе сравнения у 14 (73,7\%) установлен сколиоз 1 степени, у 5 (26,3\%) диагностирован сколиоз 2 степени. Как видно из таблицы 2, статистически значимых различий по степени тяжести сколиоза между сравниваемыми группами не получено.

Для оценки физических возможностей пациентов проводили тестирование по международной модифицированной шкале оценки функциональных возможностей Хаммерсмит (RHS), которая рекомендована для применения у детей 5 лет и старше [8, 9]. Шкала состоит из 36 пунктов, включая оценку 33 элементов в диапазоне от 0 до 2 баллов с максимально достижимой оценкой в 69 баллов. Шкала RHS является достаточно чувствительной, что позволяет оценивать степень прогрессирования заболевания и эффективность применяемых методов лечения.

В проведенном исследовании исходные функциональные возможности в двигательной сфере, рассчитанные по шкале RHS у детей в основной группе и группе сравнения, статистически не различались (табл. 2.).

Применение методов мануальной коррекции проводили с учетом степени выраженности и характера двигательных нарушений, функционального состояния мышц спины и позвоночника, индивидуальной чувствительности пациентов. Количество курсов мануального воздействия составляло от 3 до 5 курсов в год. Процедуры носили щадящий характер и не вызывали негативных реакций со стороны пациентов и их родителей.

По истечении трех лет наблюдения у детей из основной группы, в которой в лечебную стратегию были внедрены методы мануальной кор-

Характер генетических мутаций у обследованных пациентов

Таблица 1.

\begin{tabular}{ccccc}
\hline & $\begin{array}{c}\text { Число копий SMN2 } \\
\mathrm{n}=54\end{array}$ & \multicolumn{2}{c}{ Вид генетической мутации } \\
$\mathrm{n}=54$
\end{tabular}

Примечание: генотип 0/0 - гомозиготная делеция (конверсия) экзона 7 и/или 8 экзона SMN1; генотип 0 / SMN1 ${ }^{\mathrm{m}}$ - делеция на одной аллеле и внутригенная мутация на другой аллеле.

Анализ динамики показателей по категории «Балльная оценка по шкале RHS»

Таблица 2. и «Величина угла по Коббу» до и после исследования

\begin{tabular}{ccccc}
\hline Группа (СМА) & Показатель & $\begin{array}{c}\mathrm{M} \pm \text {, данные } \\
\text { до исследования }\end{array}$ & $\begin{array}{c}\mathrm{M} \pm \mathrm{S}(\%), \text { данные } \\
\text { после исследования }\end{array}$ & уровень р \\
\hline \hline Основная (СМА 2) & $\begin{array}{c}\text { Балльная оценка } \\
\text { по шкале RHS }\end{array}$ & $19,25 \pm 0,81$ & $17,02 \pm 0,28(-11,56 \%)$ & 0,0114 \\
\hline Сравнения (СMА 2) & $\begin{array}{c}\text { Балльная оценка } \\
\text { по шкале RHS }\end{array}$ & $19,16 \pm 0,74$ & $12,14 \pm 0,36(-36,73 \%)$ & $<0,0001$ \\
\hline Основная (СМА 2) & $\begin{array}{c}\text { Величина угла } \\
\text { бокового по Коббу }\end{array}$ & $6,38 \pm 0,63$ & $15,98 \pm 1,46(150,59 \%)$ & $<0,0001$ \\
\hline Сравнения (СМА 2) & Величина угла по Коббу & $6,50 \pm 0,65$ & $27,64 \pm 1,01(325,27 \%)$ & $<0,0001$ \\
\hline
\end{tabular}


рекции, отмечено замедление прогрессирования двигательных нарушений и степени тяжести сколиотической деформации позвоночника. Показатель «Балльная оценка функциональных возможностей по шкале RHS» в период «3 года» по отношению к периоду «Исходные данные» снизился для двух групп: «Основная (СМА $2) »-$ на 11,6\%, «Сравнения (СМА 2)»- на 36,7\%. Наиболее значимые изменения обнаружены для показателя «Балльная оценка функциональных возможностей по шкале RHS» группы сравнения (в среднем на 7 баллов; $\mathrm{p}<0,0001$ ) в сравнении с девиацией показателя «Балльная оценка функциональных возможностей по шкале RHS» основной группы (в среднем на 2,2; p = 0,0114) (табл. 2.).

Применение методов мануального воздействия способствовало снижению темпов прогрессирования сколиотической деформации позвоночника. Увеличение показателя «Величина деформационного угла бокового отклонения по Коббу» в период «3 года» по отношению к периоду «Исходные данные» было зарегистрировано для двух групп: «Основная (СМА 2)»- в 1,50 раза, «Сравнения (СМА 2)»- в 3,25 раза (таблица 2). О позитивном влиянии мануальной терапии свидетельствует динамика степени тяжести сколиотической деформации позвоночника: ни один пациент из основной группы не достиг 3 степени тяжести сколиоза, в то время как в группе сравнения у 19 (100\%) детей состояние ухудшилось до 3 степени тяжести.
Проведенное исследование является весьма показательным в том плане, что диагноз труднокурабельного заболевания не должен быть поводом для отказа или ограничения объема лечебных мероприятий. Своевременная диагностика, использование современных шкал для оценки двигательных возможностей пациента, применение стратегии персонифицированного подхода с включением максимально возможного спектра лечебных мероприятий способствуют замедлению темпов прогрессирования СМА с сохранением определенного объема двигательных возможностей и, соответственно, качества жизни данной категории больных детей.

\section{В Ы В О Д b}

Использование методов мануальной коррекции, включая приемы мобилизационного воздействия и постизометрической релаксации, позволяет задействовать имеющийся двигательный потенциал у детей с проксимальной формой спинальной мышечной атрофии 2 типа. Это способствует сохранению функциональных двигательных возможностей пациентов со снижением темпов прогрессирования сколиотической деформации позвоночника. Метод мануальной терапии при адекватном применении еще раз доказал свою эффективность и должен быть рекомендован для включения в программы реабилитации детей с генетически обусловленной патологией костно-мышечной системы у детей.

\section{М.Р. Шаймурзин, И.С. Луцкий, О.С. Евтушенко, М.В. Ефименко}

ГОО ВПО «Донецкий национальный медицинский университет имени М. Горького», Донецк

\section{ВОЗМОЖНОСТИ МАНУАЛЬНОЙ ТЕРАПИИ В КОРРЕКЦИИ СКОЛИОЗА У ДЕТЕЙ СО СПИНАЛЬНЫМИ МЫШЕЧНЫМИ АТРОФИЯМИ}

Актуальность. Продолжающийся поиск эффективной, безопасной терапии СМА диктует необходимость комбинаторного подхода в лечении данной патологии с определением семиотики двигательных нарушений, модификацией подходов нейрореабилитации, базирующихся на принципах ранней диагностики симптомов, влияющих на формирование осложнений и развитие тяжелой инвалидизации.

Цель. Оценить возможности мануальной коррекции сколиотических деформаций позвоночника у детей СМА.

Материал и методы. 54 ребенка со СМА 2 типа, включая 36 мальчиков (66,7\%) и 18 девочек 29 (33,3\%). Способ формирования выборки - систематический. Основная группа - 35 детей (64,8\%), у которых применялась разработанная методика лечебной физкультуры с элементами strench-гимнастики. Группа сравнения - 19 детей (35,3\%), проводилась ЛФК по общепри-

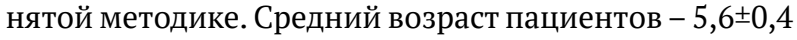

года. Пациенты оценивались по шкалам L. McPeak и RHS с фиксацией результатов в установленные отметки заболевания: «исходные данные» и «3 года».

Результаты. У детей из основной группы отмечено замедление прогрессирования двигательных нарушений и степени тяжести сколиотической деформации позвоночника: девиациия показателя «Бальная оценка функциональных возможностей по шкале RHS» основной группы в среднем на $2,2(-11,6 \%)$ балла $(\mathrm{p}<0,0001)$, снижение показателя «Бальная оценка функциональных возможностей по? шкале RHS» группы сравнения в среднем на 4,2 (в 3,4 раза) балла $(\mathrm{p}=0,0010)$. Ни один пациент из основной группы не достиг 3 степени тяжести сколиоза, в то время как в группе сравнения у 19 (100\%) детей состояние ухудшилось до 3 степени тяжести

Выводы. Предложенный комплекс проактивных реабилитационных мероприятий, направленный на коррекцию костно-суставных дефрмаций позвоноч- 
ного столба, имеет важное значение с точки зрения перспективы модификации клинического течения заболевания и улучшения качества жизни.
Ключевые слова: спинальные мышечные атрофии, манульная терапия, шкала RHS, рентгенография.

\section{M.R. Shaimurzin, I.S. Lutskii, O.S. Evtushenko, M.V. Efimenko}

\section{SEI HPE «M. Gorky Donetsk National Medical University», Donetsk}

\section{POSSIBILITIES OF MANUAL THERAPY IN CORRECTING SCOLIOSIS IN CHILDREN WITH SPINAL MUSCLE ATROPHIES}

The ongoing search for effective, safe treatment of SMA dictates the need for a combinatorial approach in the treatment of this pathology with the definition of semiotics of motor disorders, modification of neurorehabilitation approaches based on the principles of early diagnosis of symptoms that affect the formation of complications and the development of severe disability.

Purpose. To evaluate the possibilities of manual correction of scoliotic spinal deformities in children with SMA.

Material and methods. 54 children with type 2 SMA, including 36 boys (66.7\%) and 18 girls 29 (33.3\%). The sampling method is systematic. The main group - 35 children (64.8\%), who used the developed method of physiotherapy exercises with elements of strench gymnastics. Comparison group - 19 children (35.3\%); exercise therapy was carried out according to the generally accepted methodology. The average age of patients is $5.6 \pm 0.4$ years. Patients were evaluated according to the L. McPeak and RHS scales with fixing the results to the established marks of the disease: «baseline data» and «3 years».

Results. In children from the main group, there was a slowdown in the progression of motor disorders and the severity of scoliotic spinal deformity: deviation of the indicator «Ballistic assessment of functional capabilities on the RHS scale» of the main group by an average of 2.2 $(-11.6 \%)$ points $(\mathrm{p}<0.0001)$, a decrease in the «Ballistic assessment of functionality of the RHS scale» indicator of the comparison group by an average of 4.2 (3.4 times) points $(p=0.0010)$. None of the patients from the main group reached the 3rd severity of scoliosis, while in the comparison group in $19(100 \%)$ children the condition worsened to 3 severity

Conclusions. The proposed complex of proactive rehabilitation measures aimed at correcting osteoarticular deflation of the spinal column is important from the point of view of the prospects for modifying the clinical course of the disease and improving the quality of life.

Key words: spinal muscular atrophy, manul therapy, RHS scale, radiography.

\section{ЛИТЕРАТУРА}

1. Patitucci A. SMN1 gene copy number analyses for SMA healthy carriers in Italian population. J. Pediatr. Genet.2012; 1 (2): 99-102.

2. Ross L.F., Saal H.M., David K.L., Anderson R.R.; American Academy of Pediatrics; American College of Medical Genetics and Genomics. Ethical and policy issues in genetic testing and screening of children. Genet Med. 2013; 15 (3): 234-245.

3. Kwan A, Puck J.M. History and current status of newborn screeningfor severe combined immunodeficiency. Semin Perinatol. 2015; 39 (3): 194-205.

4. Zabnenkova V.V., Dadali E.L., Poljakov A.V. Analysis of the carriage of deletions in the SMN gene responsible for the occurrence of type I-IV spinal muscular atrophy. Medical Genetics. 2012. 11 (1): 3-9.

5. Забненкова В.В., Дадали Е.Л., Поляков А.В. Анализ носительства делеций в гене SMN, ответственном за возникновение спинальной мышечной атрофии I-IV типа. Медицинская генетика. 2012; 11 (1): 3-9.

6. Maretina M., Kiselev A., Zheleznyakova G., eds. Study of positive modifiers of spinal muscular atrophy severity in Russian patients. Nürnberg, Germany. 2012; 20: 334-335.

7. Committee on Bioethics, Committee on Genetics, and the American College Of Medical Genetics and the Genomics, Social,Ethical, and Legal Issues Committee. Ethical and policy issues in genetic testing and screening of children. Pediatrics. 2013; 131 (3): 620-622.

8. Dubowitz V. Spinal Muscular Atrophy Revisited. Neuromuscul. Disord. 2019; 29 (6): 413-414.

9. Соколова М.Г.Спинальная мышечная атрофия у детей: этиология, патогенез, диагностика и принципы лечения. Вестник СЗГМУ. 2013; 5 (4):108-114.

\section{REFERENCES}

1. Patitucci A. SMN1 gene copy number analyses for SMA healthy carriers in Italian population. J. Pediatr. Genet.2012; 1 (2): 99-102.

2. Ross L.F., Saal H.M., David K.L., Anderson R.R.; American Academy of Pediatrics; American College of Medical Genetics and Genomics. Ethical and policy issues in genetic testing and screening of children. Genet Med. 2013; 15 (3): 234-245.

3. Kwan A, Puck J.M. History and current status of newborn screeningfor severe combined immunodeficiency. Semin Perinatol. 2015; 39 (3): 194-205.

4. Zabnenkova V.V., Dadali E.L., Poljakov A.V. Analysis of the carriage of deletions in the SMN gene responsible for the occurrence of type I-IV spinal muscular atrophy. Medical Genetics. 2012.11 (1): 3-9.

5. Zabnenkova V.V., Dadali E.L., Polyakov A.V. An analysis of deletion carriage in the SMN gene responsible for the occurrence of type I-IV spinal muscular atrophy [Analiz nositel'stva deletsiy v gene SMN, otvetstvennom za vozniknoveniye spinal'noy myshechnoy atrofii I-IV tipa]. Medical genetics. 2012; 11 (1): 3-9.

6. Maretina M., Kiselev A., Zheleznyakova G., eds. Study of positive modifiers of spinal muscular atrophy severity in Russian patients. Nürnberg, Germany. 2012; 20: 334-335.

7. Committee on Bioethics, Committee on Genetics, and the American College Of Medical Genetics and the Genomics, Social,Ethical, and Legal Issues Committee. Ethical and policy issues in genetic testing and screening of children. Pediatrics. 2013; 131 (3): 620-622.

8. Dubowitz V. Spinal Muscular Atrophy Revisited. Neuromuscul. Disord. 2019; 29 (6): 413-414.

9. Sokolova M.G. Spinal muscular atrophy in children: eti- 
10. Aleksiev A.R. A novel physical therapy method of treating myofascial pain due to muscle spasm and shortening. Folia Med (Plovdiv). 2013; 55 (2):43-50.

11. Darras B.T. Spinal muscular atrophies. Pediatr. 2015; 62 (3): 743-766.

12. Ogino S., Wilson R.B. Spinal muscular atrophy: molecular genetics and diagnostics. Expert Rev. Mol. Diagn. 2004; 4 (1): 15-29.

13. Chien Y.H., Chiang S.C., Weng W.C., eds. Presymptomatic diagnosis of spinal muscular atrophy through newborn screening. J Pediatr. 2017; 190: 124-1294.

14. Wirth B. Moving towards treatments for spinal muscular atrophy: hopes and limits. Expert Opin. Emerg. Drugs. 2015; 20 (3): 353-6.

15. Hamilton G, Gillingwater T.H. Spinal muscular atrophy: going beyond the motor neuron. Trends Mol Med. 2013; 19 (1): 40-50.

16. Botkin J.R., Belmont J.W., Berg J.S., eds. Points to consider: ethical,legal, and psychosocial implications of genetic testing in children and adolescents. Am J Hum Genet. 2015; 97 (1): 6-21. ology, pathogenesis, diagnosis and treatment principles [Spinal'naya myshechnaya atrofiya u detey: etiologiya, patogenez, diagnostika i printsipy lecheniya]. Bulletin of SZGMU. 2013; 5 (4): 108-114.

10. Aleksiev A.R. A novel physical therapy method of treating myofascial pain due to muscle spasm and shortening. Folia Med (Plovdiv). 2013; 55 (2):43-50.

11. Darras B.T. Spinal muscular atrophies. Pediatr. 2015; 62 (3): 743-766.

12. Ogino S., Wilson R.B. Spinal muscular atrophy: molecular genetics and diagnostics. Expert Rev. Mol. Diagn. 2004; $4(1): 15-29$.

13. Chien Y.H., Chiang S.C., Weng W.C., eds. Presymptomatic diagnosis of spinal muscular atrophy through newborn screening. J Pediatr. 2017; 190: 124-1294.

14. Wirth B. Moving towards treatments for spinal muscular atrophy: hopes and limits. Expert Opin. Emerg. Drugs. 2015; 20 (3): 353-6.

15. Hamilton G, Gillingwater T.H. Spinal muscular atrophy: going beyond the motor neuron. Trends Mol Med. 2013; 19 (1): 40-50.

16. Botkin J.R., Belmont J.W., Berg J.S., eds. Points to consider: ethical,legal, and psychosocial implications of genetic testing in children and adolescents. Am J Hum Genet. 2015; 97 (1): 6-21. 